\title{
A Fingerprint Pointing Device Utilizing the Deformation of the Fingertip during the Incipient Slip
}

\author{
Yuichi Kurita, Atsutoshi Ikeda, Jun Ueda, and Tsukasa Ogasawara, Member, IEEE
}

\begin{abstract}
In this paper, a novel pointing device is proposed that utilizes the deformation of the fingertip. When a fingertip is pressed and slightly slid on a rigid plate, a partial slip, called an "incipient slip", occurs on the contact surface. While the deformation around the center of the contact area is small during the incipient slip, the boundary region moves to the sliding direction of the fingertip. The deformation changes depending on the sliding distance of the fingertip and the exerted force on the contact surface. The velocity of the pointer can be determined by the estimated distance and force based on the measurement of the deformation.

In this study, the correlation between the sliding distance of the fingertip and the deformation, and between the exerted force and the deformation are investigated. The degree of the deformation due to the sliding motion can be estimated based on the detected fingerprint center. The Group Delay Spectrum (GDS) tracking method is proposed for the detection of the fingerprint center. A prototype pointing device is developed to evaluate the operationality of the proposed device. Comparative experiments with conventional pointing devices are conducted. The validity of the proposed device is confirmed by the experiments.
\end{abstract}

Index Terms-Pointing device, fingerprint deformation, incipient slip, group delay spectrum

\section{INTRODUCTION}

$\mathbf{I}$ MPROVEMENT in hardware technology allows the continued miniaturization of computer hardwares. Likewise, the performance of PDAs (Personal Digital Assistants) and mobile phones have also improved year by year. To operate these compact information devices, input devices should be small and easy to use. Conventional pointing devices, however, such as computer mice, slide pads, and track points, have disadvantages in operationality because of their size for portable devices.

On the one hand, a number of man-machine interface systems have been proposed using human's eye motions[1], [2], gesture recognition of an operator's hands and fingers[3],

Manuscript received January 20, 2002; revised November 18, 2002. This work was supported in part by 21 st Century COE Program "Ubiquitous Networked Media Computing" and in part by a Grant-in-Aid for Scientific Research B(2) (No. 13450104), MEXT, Japan.

Yuichi Kurita is with the Graduate School of Engineering, Hiroshima University, 1-4-1 Kagamiyama, Higashi-Hiroshima, Hiroshima, 739-0045, Japan (E-mail: kurita@hfl.hiroshima-u.ac.jp).

Atsutoshi Ikeda is with Yamaha Motor Co., Ltd., 2500 Shingai, Iwata, Shizuoka, Japan.

Jun Ueda and Tsukasa Ogasawara are with the Graduate School of Information Science, Nara Institute of Science and Technology (NAIST), 89165 Takayama, Ikoma, Nara, 630-0192, Japan (E-mail: uedajun@is.naist.jp, ogasawar@is.naist.jp).
[4], and other features[5], [6], [7]. These vision-based interfaces have advantages in that the operator's motions can be measured without any attachments to his/her body. On the other hand, wearable measuring systems have also been proposed such as wearable gloves[8], [9], rings[10], or special devices attached on the arm[11] and nails[12]. However, these complex systems are not suitable for portable devices.

In order to develop a novel pointing device that has good operationality, occupies a small space, and is low in cost, we propose to use the deformation of the fingertip during the incipient slip. An operator presses the fingertip and slightly slides it over the surface of the fingerprint-capturing device. During such motion, a partial slip occurs on the contact surface before the finger completely slips relative to the surface. This phenomenon, called "incipient slip" occurs because the pressure is smaller around the boundary of the contact region than around the center[13], [14]. By measuring and analyzing the incipient slip, the movement of the fingertip can be estimated even in the slight sliding motion of the fingertip. Since the gross slip is unnecessary for the operation of the pointer, operators can control the pointing device with a minimum fingertip motion. In addition, a click action can also be detected by measuring the area of the contact region, which expands as the strength of the pressure increases.

Fingerprint images can be used for a fingerprint verification. Therefore, the fingerprint capturing device can be shared with the proposed pointing device. The proposed pointing device can be miniaturized since very small and thin fingerprint capturing devices have been developed.

In this paper, the characteristics of the fingertip deformation is quantitatively investigated. Based on the investigation, a novel operational method for pointing devices that use the deformation of the fingertip is proposed. Additionally, a prototype device is developed to evaluate the operationality of the proposed method.

\section{Fingertip DEFormation DURING INCIPIENT SLIP}

\section{A. Incipient Slip}

A human's fingertip roughly consists of a bone, subcutaneous tissue, and skin. When a fingertip is pressed and slid on a rigid plate, the deformation of the fingertip can be assumed as the deformation of an elastic sphere. Since the pressure around the boundary of the contact region is smaller than the pressure of the center, the slip between the sphere and the plate occurs in the boundary region. If the entire contact 
region slips, the sphere begins to slip relative to the plate. The partial slip of an elastic object, which occurs before the gross slip, is called an "incipient slip". This phenomenon can be approximately expressed in Fig.1. Johansson et al. have shown that perceiving the incipient slip is important for human's sophisticated grasp[15]. In recent years, a number of tactile sensors have been proposed, e.g., for detecting the contact position[16], [17], the slippage[18], [19], [20], and for distinguishing the difference of the friction coefficient[21], [22]. In order to examine the stick/slip state of the human's fingertip during the incipient slip, a fingerprint measurement system is developed. The overview is shown in Fig.2(a). A subject puts the hand on the table of the system and presses the index finger on the fingerprint capturing device (BMF Corporation, EZF-650, a pressure reaction type). The subject's hand is fixed on the table by a rubber belt and the index finger is pinched on both sides. The fingerprint capturing device is fixed on a 3-axes stage so that the position of the sensor surface can be adjusted. Hereafter, the coordinate axes are defined as shown in Fig.2(b).

Five subjects volunteered for the experiments. The representative examples of the experimental results are shown in Fig.3. Fig.3(e) indicates the captured image at the neutral position, and Fig.3(a) (d) indicate the images when the stage is moved to the negative direction of the $\mathrm{X}$-axis for $1[\mathrm{~mm}] \sim 4[\mathrm{~mm}]$ respectively. Fig.4 shows the differential images between Fig.3(e) and Fig.3(a) (d) respectively. We can observe the slip region expands from the boundary toward the center of the contact region. When the sensor surface is moved more than $4[\mathrm{~mm}]$, almost the entire contact region becomes the slip region.

\section{B. Pointing Device Utilizing the Deformation of the Fingertip}

In this paper, a control method of a pointing device is proposed based on the deformation of the fingertip. An operator can control the pointing device by a small motion of the fingertip because the incipient slip occurs before the fingertip is completely slipped on the sensor surface.

Hereafter we define the sliding motion before the fingertip is completely slipped as a "semi-sliding motion". In the semisliding motion, the sliding distance is approximately less than $4[\mathrm{~mm}]$. During the incipient slip, the stick region remains around the center of the contact region. Furthermore, the contact region moves toward the direction of the fingertip motion. If the center of the contact region can be tracked,

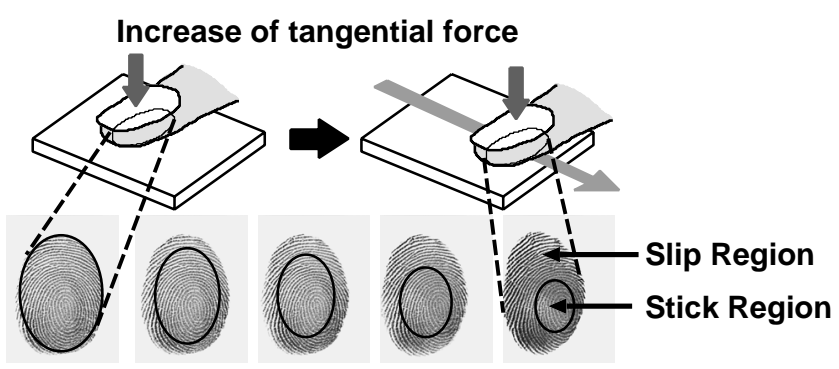

Fig. 1. Diagram of an incipient slip.

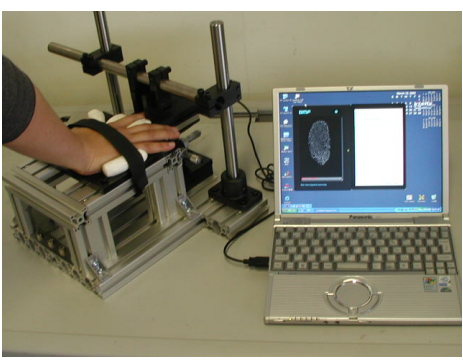

(a)

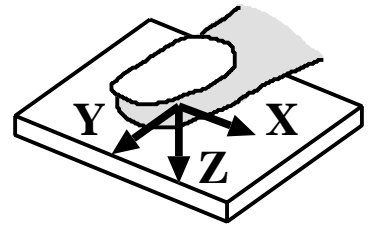

(b)
Fig. 2. Fingerprint measurement system. (a) System overview. (b) Coordinate axes.

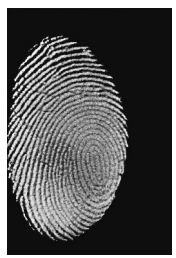

(a)

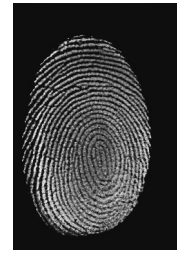

(b)

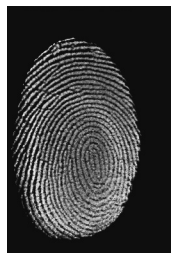

(c)

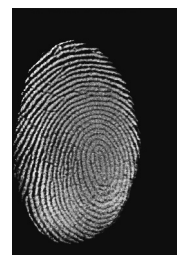

(d)

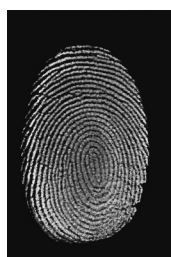

(e)

Fig. 3. Captured images during the sliding motion of a fingertip. The captured image size is $256 \times 384$ [pixel]. (a) The sliding distance is 1 [mm]. (b) $2[\mathrm{~mm}]$. (c) $3[\mathrm{~mm}]$. (d) $4[\mathrm{~mm}]$. (e) Image at the neutral position.

the moving direction and the distance of the fingertip can be estimated. The pointer velocity of the pointing device can be controlled based on the estimated direction and the distance of the operator's small fingertip motion. In addition, the contact region expands when an operator strongly presses the fingertip on the sensor surface. A click action can also be detected by calculating the change of the contact area.

Mascaro et al. have developed a photoplethysmograph fingernail sensor that can optically measure the pattern of blood volume beneath the fingernail and predict the normal force, shear force, and finger posture[23], [24]. The proposed device is similar to the fingernail sensor on the point that the devices can measure the fingertip deformation and be utilized as a man-machine interface; however the measurement method of the deformation is completely different. Moreover, the proposed device is suitable for portable devices because the device is small and can be shared with a verification system.

\section{Eccentricity of the Contact Area}

A number of researchers have investigated the deformation of the fingertip during the contact and slippage[25], [26], [27], [28]. In this section, the characteristics of the fingertip deformation are investigated based on the eccentricity of the contact area. Fig.5(a) shows the fingerprint image of the index 


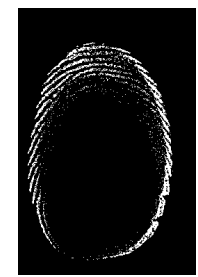

(a)

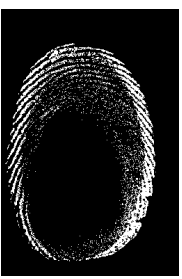

(b)

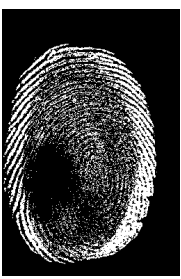

(c)

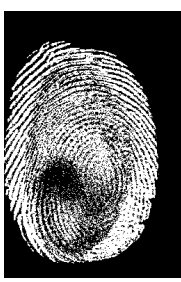

(d)
Fig. 4. Differential between the image during the sliding motion and the image at the neutral position. (a) The sliding distance is $1[\mathrm{~mm}]$. (b) $2[\mathrm{~mm}]$. (c) $3[\mathrm{~mm}]$. (d) $4[\mathrm{~mm}]$.

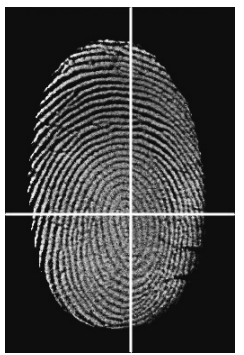

(a)

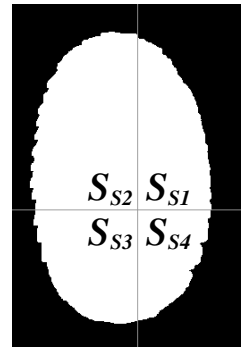

(b)
Fig. 5. Segmentation of the contact area. (a) Original image. (b) Binarized image.

finger at the neutral position. Fig.5(b) shows the binarized image at a certain threshold after being smoothed using a $7 \times 7$ filter. The intersection of the horizontal and the vertical lines indicates the fingerprint center determined previously. The fingerprint images when the fingertip is slid along $\mathrm{X}$-axis are also shown in Fig.6. The fingerprint image is divided into four quadrants based on the fingerprint center. Each quadrant area is defined as $S_{s 1}, S_{s 2}, S_{s 3}$ and $S_{s 4}$ respectively. Similarly, each quadrant area after the semi-sliding motion is defined as $S_{t 1}, S_{t 2}, S_{t 3}$ and $S_{t 4}$. The eccentricity $e_{x}, e_{y}$ and $e_{z}$ are defined as follows:

$$
\begin{aligned}
e_{x} & \triangleq \frac{S_{t x}}{S_{t}}-\frac{S_{s x}}{S_{s}} \\
e_{y} & \triangleq \frac{S_{t y}}{S_{t}}-\frac{S_{s y}}{S_{s}} \\
e_{z} & \triangleq S_{t}-S_{s}
\end{aligned}
$$

where $S_{s}$ is the entire contact area at the neutral position, and $S_{t}$ is the entire contact area after the fingertip moves. The change in the contact area is divided by the entire contact

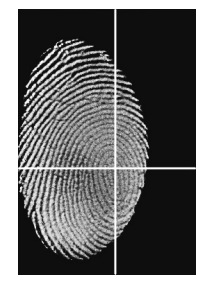

(a)

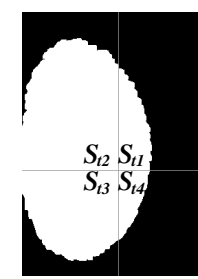

(b)

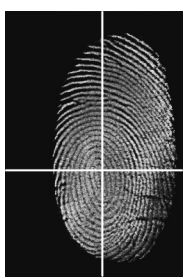

(c)

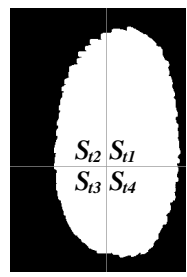

(d)
Fig. 6. Deformation of the contact area. (a) Left (original). (b) Left (binarized). (c) Right (original). (d) Right (binarized).

area for the normalization. The area difference at the neutral position along the $\mathrm{X}$-axis $S_{s x}$ and along the Y-axis $S_{s y}$ are defined as follows:

$$
\begin{aligned}
& S_{s x} \triangleq\left(S_{s 1}+S_{s 4}\right)-\left(S_{s 2}+S_{s 3}\right) \\
& S_{s y} \triangleq\left(S_{s 3}+S_{s 4}\right)-\left(S_{s 1}+S_{s 2}\right)
\end{aligned}
$$

Similarly, the area difference after the semi-sliding motion along the X-axis $S_{t x}$ and along the Y-axis $S_{t y}$ are defined.

If the fingertip is assumed as an elastic sphere, we have the following approximation between the eccentricity $e_{x}$ and the sliding distance $\delta_{x}$ along the $\mathrm{X}$-axis (see Appendix ) :

$$
e_{x} \cong \frac{4 \delta_{x}}{\pi a}
$$

where $a$ is the radius of the contact area. This equation indicates that the eccentricity is in proportion to the sliding distance.

\section{Characteristics of the Fingerprint Deformation}

In order to investigate the correlation between the eccentricity and the fingerprint deformation, the correlation between the eccentricity and the sliding distance of the fingertip, and the correlation between the eccentricity and the exerted force on the sensor surface are examined.

The measurement system and subjects are the same as described in the section II-A. A subject puts the index finger on the fingerprint capturing device and presses it at $9.8[\mathrm{~N}]$. The fingerprint images and the exerted force are measured at every $0.5[\mathrm{~mm}]$ shift of the stage along the $\mathrm{X}, \mathrm{Y}$ and $\mathrm{Z}$-axis.

Fig.7(a), (c) and (e) indicate the correlation between the eccentricity and the sliding distance. Fig.7(b), (d) and (f) indicate the correlation between the eccentricity and the exerted force along the $\mathrm{X}, \mathrm{Y}, \mathrm{Z}$-axis. The approximate lines are also shown in these figures.

We can observe the linear correlation between the eccentricity and the sliding distance, and between the eccentricity and the exerted force along X-axis. Moreover, the linear correlation between the eccentricity and the sliding distance is stronger than that between the eccentricity and the exerted force. The averages of $R^{2}$ values from the approximate lines on each subject are shown in Table I. $R^{2}\left(0<R^{2}<1\right)$ is the determination coefficient and the approximation is good when the value is large. The variation of the data is also shown in Table II. The variation coefficient can be calculated based on the division of the standard deviation by the average. The variation of data is large when the variation coefficient is large.

These experimental results show that the eccentricity has a linear correlation with the sliding distance of the fingertip. Therefore, the pointer can be controlled by the estimation of the sliding distance based on the measurement of the eccentricity.

TABLE I

Average of $R^{2}$

\begin{tabular}{l|ll}
\hline \hline axis & distance & force \\
\hline $\mathrm{X}$ & 0.99 & 0.96 \\
$\mathrm{Y}$ & 0.89 & 0.82 \\
$\mathrm{Z}$ & 0.87 & 0.55 \\
\hline
\end{tabular}

TABLE II

VARIATION COEFFICIENTS

\begin{tabular}{l|ll}
\hline \hline axis & distance & force \\
\hline $\mathrm{X}$ & 0.11 & 0.27 \\
$\mathrm{Y}$ & 0.47 & 0.51 \\
$\mathrm{Z}$ & 0.41 & 0.61 \\
\hline
\end{tabular}




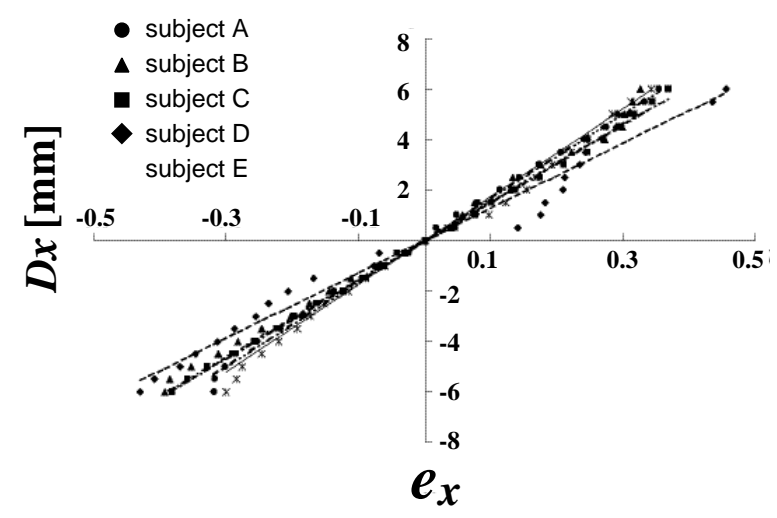

(a)

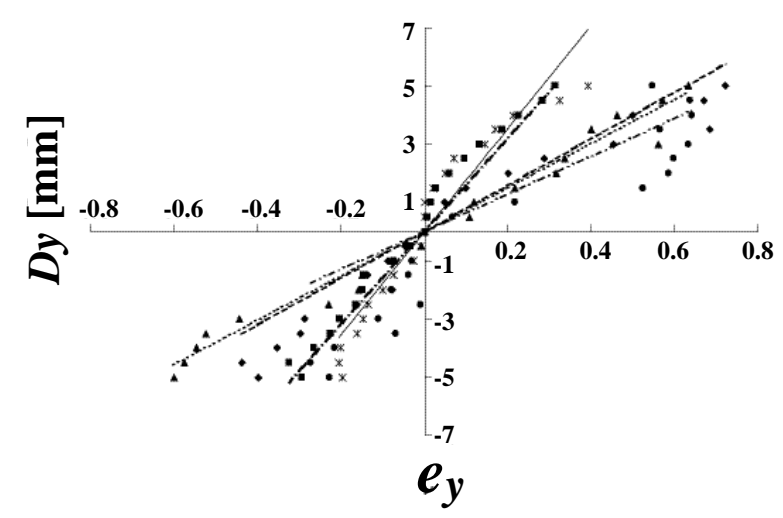

(c)

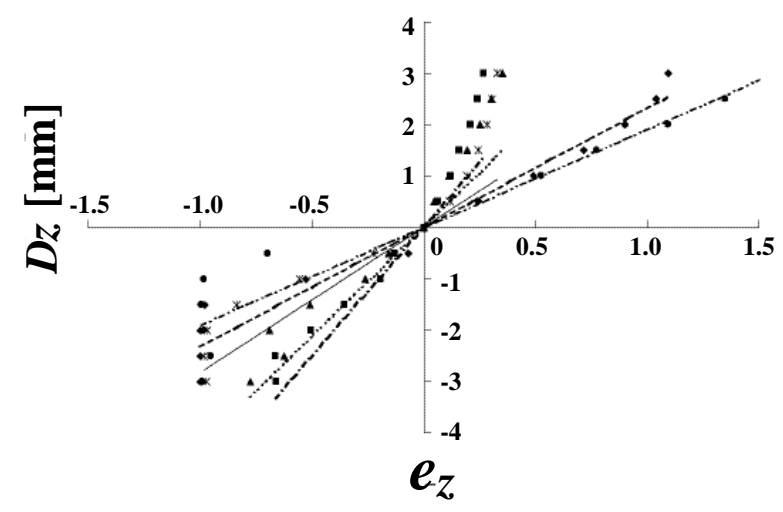

(e)

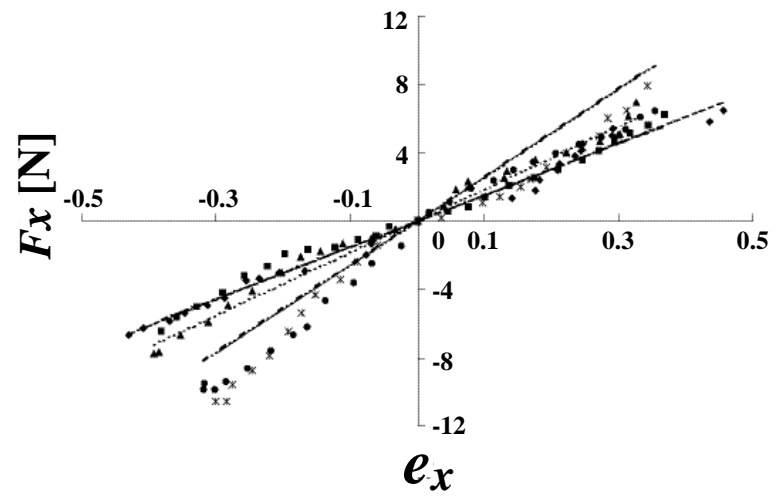

(b)

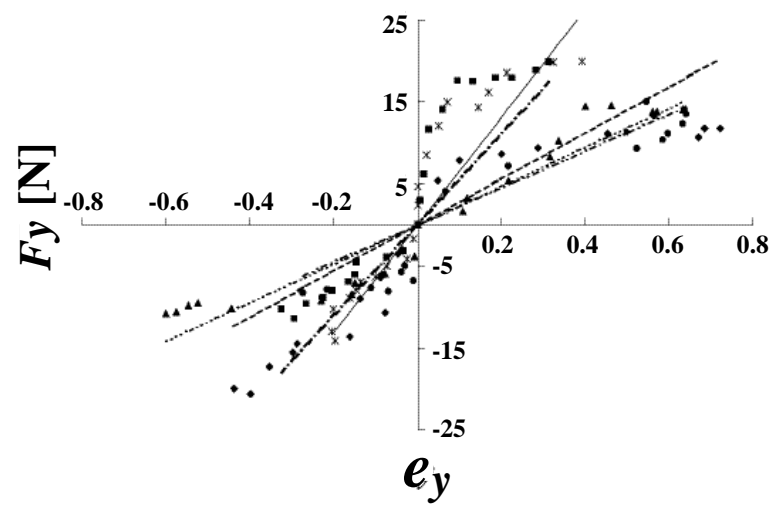

(d)

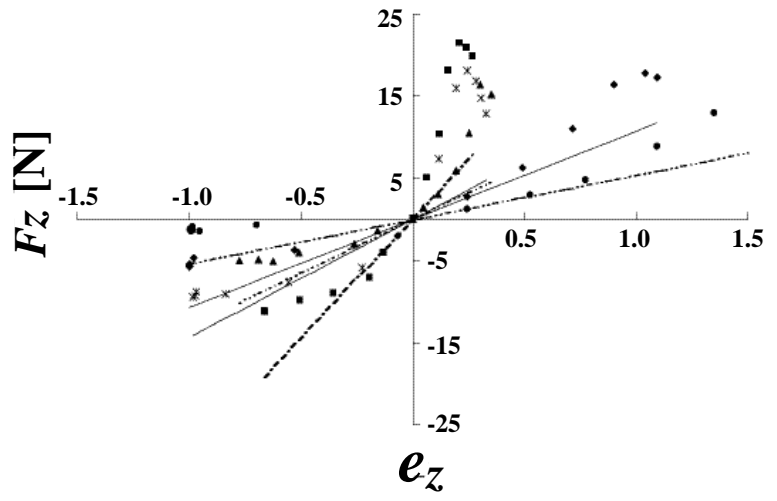

(f)

Fig. 7. Correlation between the eccentricity and the distance, and between the eccentricity and the exerted force. (a) Eccentricity and distance (X-axis). (b) Eccentricity and force (X-axis). (c) Eccentricity and distance (Y-axis). (d) Eccentricity and force (Y-axis). (e) Eccentricity and distance (Z-axis). (f) Eccentricity and force (Z-axis).

\section{Determination OF THE DiRECTION AND Velocity OF THE POINTER}

\section{A. Detecting the Center of the Fingerprint}

When a gross slip occurs on a sensor surface, the center of the fingerprint shifts according to the slippage. In order to calculate the eccentricity under an unexpected slippage, the center of the fingerprint should be tracked during the motion.

To detect the center of the fingerprint, fingerprint verification methods[29], such as the minutiae verification[30], and template matching[31] can be used. However, these methods require a high calculation time in the detection when the quality of the fingerprint image is not high. While these methods are effective for the verification, it is difficult to adapt to a real-time processing system such as a pointing device.

To overcome this problem, we propose to utilize the group delay spectrum (GDS)[32], [33] to detect and track the center of the fingerprint. Since the proposed GDS tracking method is calculated using one dimensional time-line signals transposed from a row or column line of the fingerprint image, the calculation time for the detection of the fingerprint center 


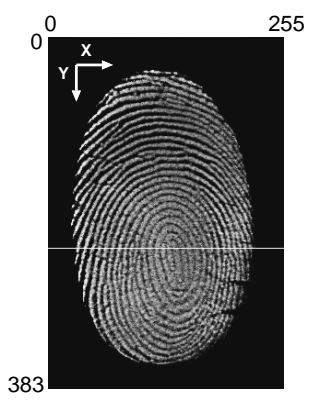

(a)

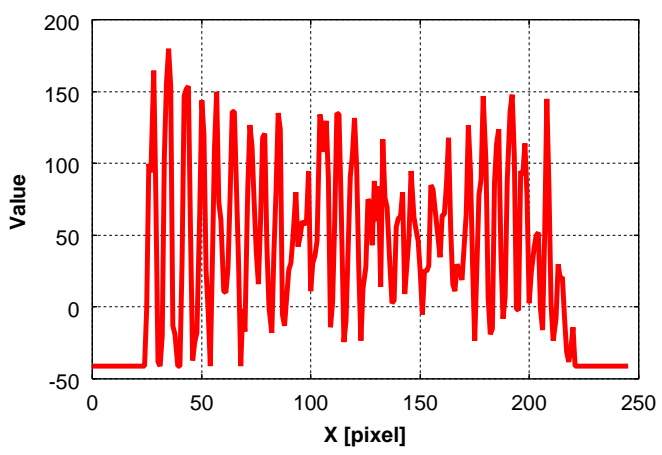

(b)

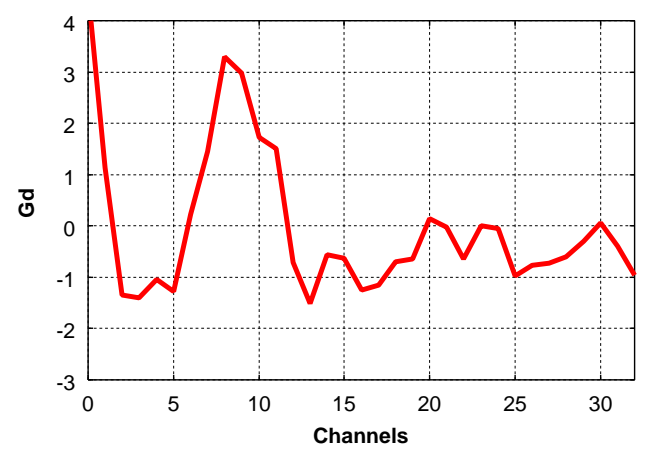

(c)

Fig. 8. GDS analysis where the order of the prediction $p$ is set 32. (a) Fingerprint image captured in the size of $256 \times 384$ [pixel]. (b) Waveform on the white line of (a). (c) GDS of (b).

relatively takes a short time. Furthermore, the spectrum is robust to noises because the spectrum peaks are emphasized in the GDS.

\section{B. GDS Analysis}

The GDS is defined as the frequency differential of the phase spectrum of a polar form filter $H(z)$, which is estimated by a linear prediction method. $H(z)$ is defined as the following equation:

$$
H(z)=\frac{1}{1+\sum_{k=1}^{p} a_{k} z^{-k}}
$$

where $a_{k}$ is the coefficient of the linear prediction and $p$ is the order of the prediction.

The phase spectrum $\theta\left(\omega_{i}\right)$ of $H(z)$ is derived from the following equations:

$$
\begin{aligned}
\theta\left(\omega_{i}\right) & =-\tan ^{-1}\left[\frac{\operatorname{Im}\left\{H^{\prime}\left(\omega_{i}\right)\right\}}{\operatorname{Re}\left\{H^{\prime}\left(\omega_{i}\right)\right\}}\right] \\
H^{\prime}\left(\omega_{i}\right) & =1+\sum_{k=1}^{p} a_{k} e^{-k j \omega_{i} T}
\end{aligned}
$$

where $T$ is the sampling period, $\omega_{i}=(\pi / L) i \quad(i=$ $1,2, \cdots, L)$, and $L$ is the division number of the frequency range.

Accordingly, the group delay spectrum $T_{g}\left(\omega_{i}\right)$ is given by the following equation:

$$
T_{g}\left(\omega_{i}\right)=-\frac{\Delta \theta\left(\omega_{i}\right)}{\Delta \omega}
$$

where $\Delta \theta\left(\omega_{i}\right)=\theta\left(\omega_{i}\right)-\theta\left(\omega_{i-i}\right)$, and $\Delta \omega=\pi / L$.

To adapt to a noisy image, the coefficient of the linear prediction $a_{k}$ is weighted for smoothing the peaks of the GDS:

$$
a_{k}^{\prime}=c^{k} a_{k} \quad(0 \leq c \leq 1)
$$

The example of the GDS analysis is shown in Fig.8.

\section{GDS Tracking Method}

The schematic diagram for the detection the fingerprint center is shown in Fig.9. Before the operation, the coordinate of the center $\left(x_{t}, y_{t}\right)$ is registered from the fingerprint image at the neutral position. Next, the row and column lines that include the coordinate $\left(x_{t}, y_{t}\right)$ are analyzed by the GDS analysis. The resultant GDS are stored as $\boldsymbol{g}_{x}^{p \times 1}$ and $\boldsymbol{g}_{y}^{p \times 1}$.

For row lines, the following value $d_{i}$, which is the total Euclidean norm in the range of $R$, is calculated:

$$
d_{i} \triangleq \sum_{i-R}^{i+R}\left|\boldsymbol{g}_{i}-\boldsymbol{g}_{x}\right|
$$

where $\boldsymbol{g}_{i}$ is the GDS at the $i$-th line during the operation.

Considering that the center of the fingerprint consecutively moves, the evaluation value $d_{i}^{\prime}$ is determined depending on the distance between the currently searched line $i$ and the previously detected line $i^{\prime}$ that includes the center:

$$
d_{i}^{\prime} \triangleq W_{i} d_{i}
$$

where $W_{i}=\left|i^{\prime}-i\right| / 3+1$.

Finally, the coordinate of the fingerprint center $i^{*}$ is detected by:

$$
i^{*}=\operatorname{argmin}\left(d_{1}^{\prime}, \ldots, d_{N_{w i d t h}}^{\prime}\right)
$$

where $N_{\text {width }}$ is the width of the image.

For the Y-direction, the coordinate of the fingerprint center $j^{*}$ is detected in the same way. As a result, the coordinate of the center is detected as $\left(i^{*}, j^{*}\right)$. 


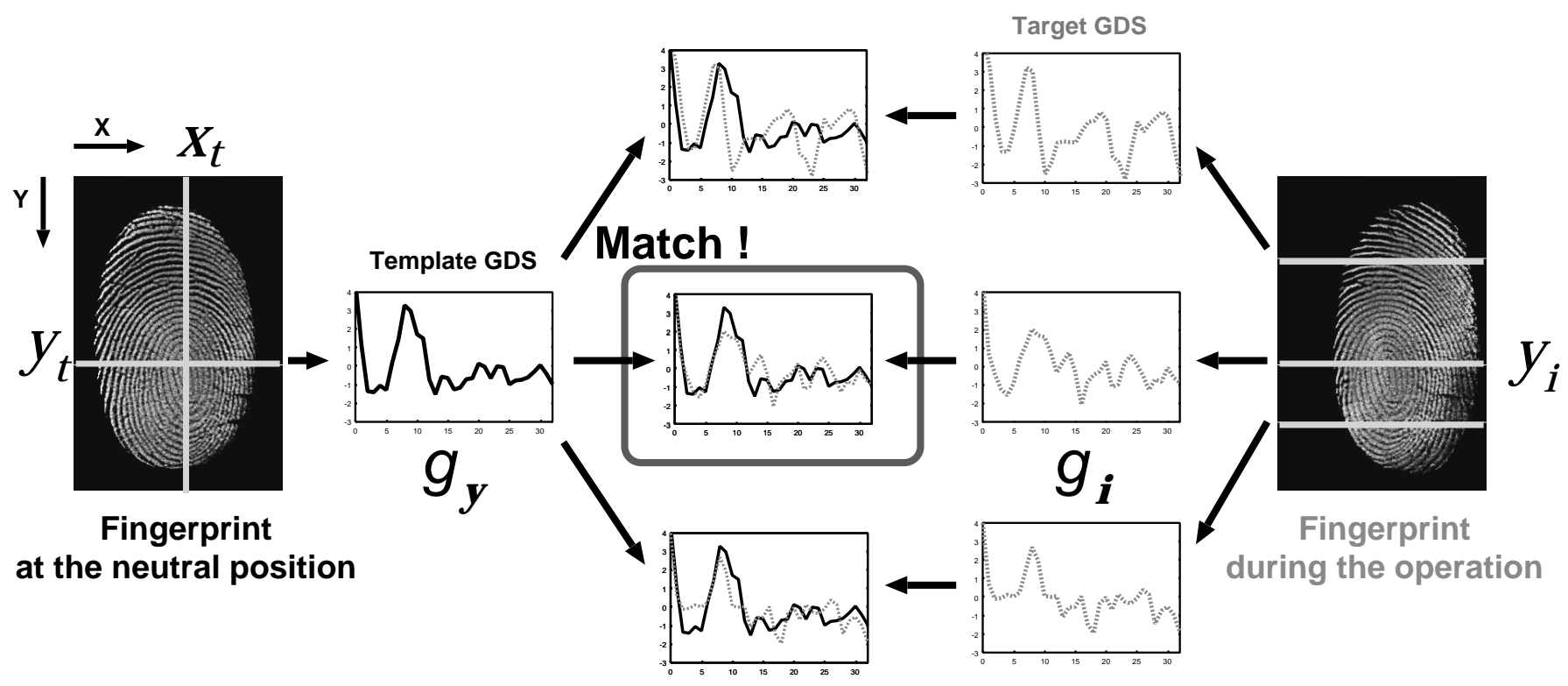

Fig. 9. Schematic diagram of the GDS tracking method. $\left(x_{t}, y_{t}\right)$ is the registered coordinate of the fingerprint center and $y_{k}$ is the currently searched line. $g_{y}$ and $g_{i}$ are the GDS of the $y_{t}$-th and $y_{i}$-th lines respectively. all the $g_{i}$ are compared with $g_{y}$ and the most similar line is detected.

\section{Validity of the GDS Tracking Method}

The GDS of row and column lines, which are registered as the template, should be selected where the signals are not largely affected by the fingerprint deformation during the semisliding motion. In order to select the adequate point for the template, the effect of the fingerprint deformation on the GDS is investigated.

The experimental procedure for row lines are shown in Fig.10. At first, the norm $d_{i}^{j}$ at the $i$-th line $(i=$ $\left.0 \cdots N_{\text {height }}\right)$ between the neutral image and the $j$-th target image $(j=1 \cdots K)$ is calculated from Eq.(12). Next, the vector $\boldsymbol{r}_{\text {row }}^{N_{\text {heigh }} \times 1}$ is defined from the average of $\boldsymbol{r}_{j}$ :

$$
\boldsymbol{r}_{\text {row }} \triangleq \frac{\sum_{j=1}^{K} \boldsymbol{r}_{j}}{K}
$$

where $\boldsymbol{r}_{j}^{N_{\text {height }} \times 1}$ is the vector consists of $d_{i}^{j}$.

One fingerprint image at the neutral position and 12 images during the semi-sliding motion $(K=12)$ to the positive and negative directions along the $\mathrm{X}$ and Y-axes (three images for each direction) are used for the investigation. Fig.11(a) indicates $\boldsymbol{r}_{\text {row }}$ after being smoothed by the six neighborhoods where $R=5$. The result for the column lines $\boldsymbol{r}_{c o l}$ is also shown in Fig.11(b). The area where the norm is less than 1000 for the row lines and 1150 for the column lines is shown in Fig.11(c). It can be observed the small norm is distributed around the center of the fingerprint. Since the fingerprint deformation around the center is smaller than that around the boundary region, the GDS tracking method has successfully detected and tracked the center of the fingerprint during the semi-sliding motion.

\section{E. Pointer Velocity and Click Action}

As described in the section II-D, the eccentricity has a linear correlation with the sliding distance of the fingertip.

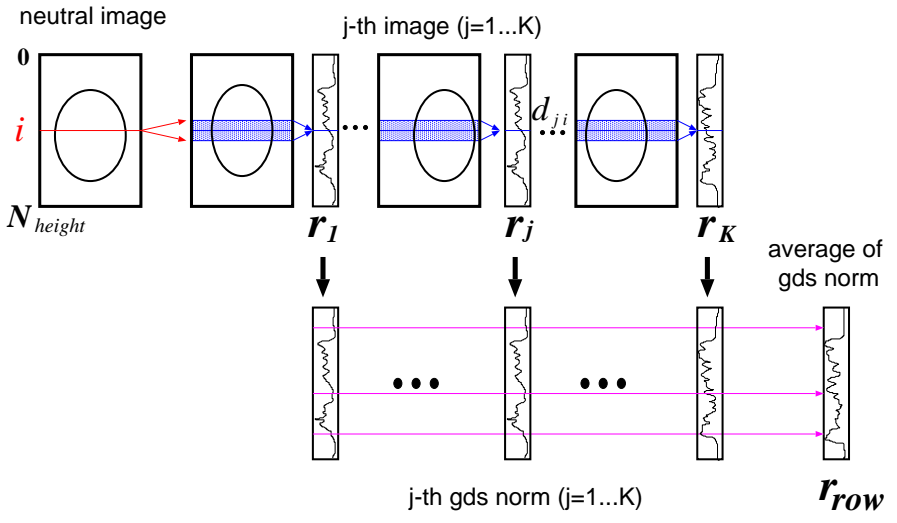

Fig. 10. Validity evaluation method of the GDS tracking.

The pointer velocity $\Delta X$ and $\Delta Y$ is determined based on the average of the approximate lines $K_{x}$ and $K_{y}$ :

$$
\begin{aligned}
\Delta X & =M_{x} K_{x} e_{x} \\
\Delta Y & =M_{y} K_{x} e_{y}
\end{aligned}
$$

where the $M_{x}$ and $M_{y}$ are the moderator variables.

Fig.12 shows the binarized images at a certain threshold after being smoothed using a $7 \times 7$ filter in the pushing motion. When the fingertip is pressed on the sensor surface, the contact area expands as the strength of the pressure increases. The click action can be detected when the whole contact area exceeds a certain threshold.

\section{IMPLEMENTATION}

\section{A. System Configuration}

A prototype of the proposed pointing device system is developed. The overview of the developed system is shown 


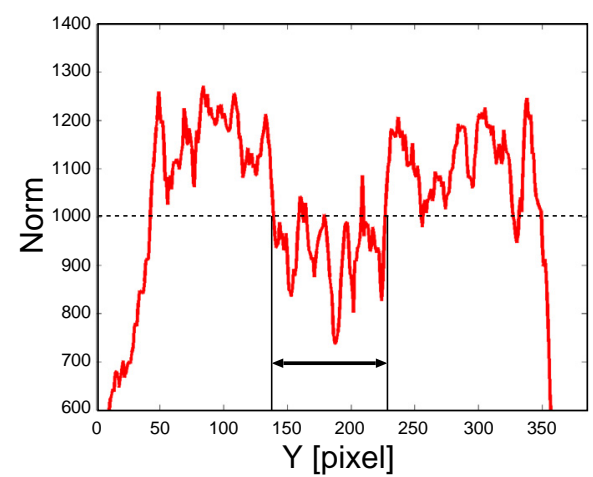

(a)

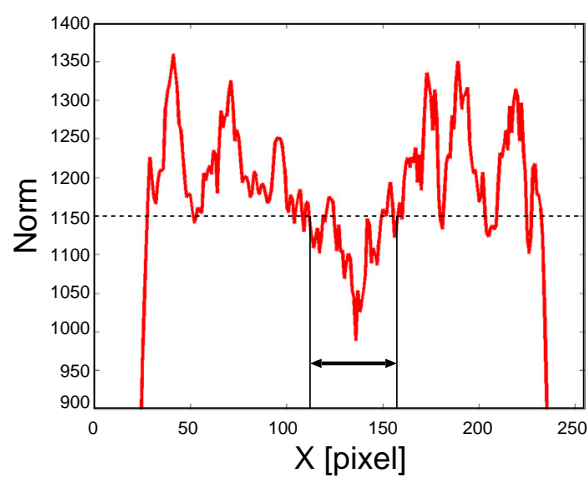

(b)

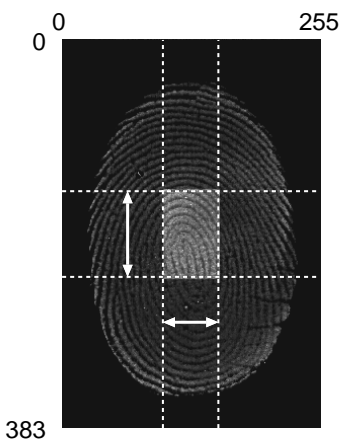

(c)

Fig. 11. Validity of the GDS tracking method. The contact area between the fingertip and the sensor surface is about $30<X<220,50<Y<330$. (a) GDS norm for the row line. (b) GDS norm for the column line. (c) The area where the norm is less than 1000 for the row lines and 1150 for the column lines.

in Fig.13(a). Fingerprint images are captured by an electric capacitance-type sensor (Fujitsu Microelectronics Europe, BMF110-PWF1) shown in Fig.13(b). Fig.14 shows the captured fingerprint image. The sensing area is $15 \times 15[\mathrm{~mm}]$ and the resolution of the captured image is $300 \times 300$ [pixel]. The sampling time changes depending on the resolution of the captured image and the sampling frequency is about $54[\mathrm{~Hz}]$ and $14[\mathrm{~Hz}]$ respectively.

The captured fingerprint images are transferred to a computer (Pentium III 500[MHz], Linux 2.4.18) via ISA bus. The center of the fingerprint is tracked by the GDS tracking method. The processing time for the GDS tracking also

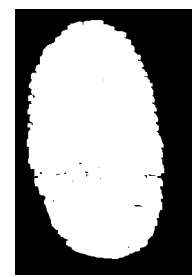

(a)

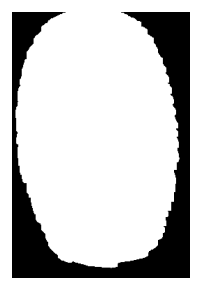

(b)
Fig. 12. Binarized image during the pushing motion. (a) Before pushing. (b) After pushing.

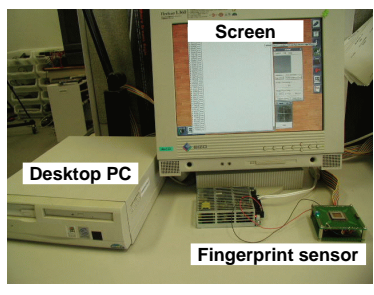

(a)

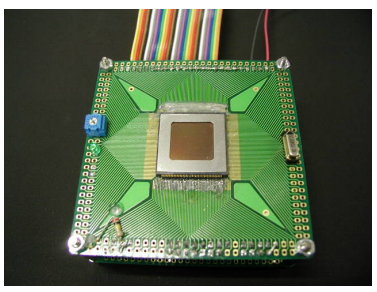

(b)
Fig. 13. Prototype of the pointing device system. (a) System overview. (b) Fingerprint sensor.

changes depending on the resolution of the image. When the image resolution is $60 \times 60$ [pixel] and $120 \times 120$ [pixel], the processing time are about $30[\mathrm{msec}]$ and $180[\mathrm{msec}]$. When the image resolution is $60 \times 60$ [pixel] and $120 \times 120$ [pixel], the total detecting speed is about $50[\mathrm{~Hz}]$ and $8[\mathrm{~Hz}]$ respectively.

In the experiment, the resolution of the fingerprint images is set $60 \times 60$ [pixel]; the order of the linear prediction $p$ is set 16 ; the division number of the frequency range $L$ is set 16 ; the weight coefficient of the linear prediction $c$ is set 0.9 ; the range of the total GDS norm is set $R=0$; the coefficient $K_{x}$ and $K_{y}$ are set 15.5 and 11.1 based on the results in the section III-E, and the moderator variables of the pointer velocity $M_{x}$ and $M_{y}$ are set 0.5 and 0.8 respectively.

\section{B. Registration of the Fingerprint Center}

Before the experiment, the center of the fingerprint at the neutral position is registered. The fingerprint center was manually set where it seemed to be unique for the subject. The subject put the fingertip so that the fingerprint center was located at the center of the sensor surface and registered the center by watching the screen.

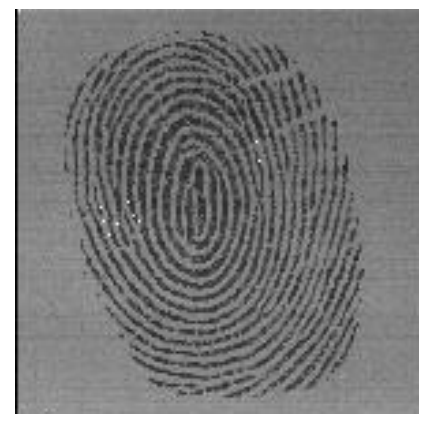

Fig. 14. Fingerprint image captured by the developed system. 
Each subject is required to register the fingerprint center once before the operation. In this experiment, the subjects manually registered the center, but an automatic registration tool has been developed that can register the fingerprint center when the subject puts the fingertip on the sensor surface.

\section{Experimental Result}

A subject operates the pointer on $\mathrm{X}$ Window System of Linux using the developed pointing device. The experimental scene is shown in Fig.15.

In order to evaluate the operationality of the developed pointing device, a performance test was conducted where the subject controls the pointer and clicks the circles on the screen. The subject sequentially moved the pointer to six circles with a radius of 20 [pixel] that was drawn on the $500 \times 500$ [pixel] window using the proposed system, a touch pad, and a pointing stick mounted on a notebook computer. The representative trajectory of the pointer and the click positions are shown in Fig.16.

The subject operates the pointer on the screen by sliding the fingertip slightly on the sensor surface. The developed system measures the eccentricity of the fingerprint and the pointer is controlled by the velocity control. A touch pad measures the position of the fingertip on the sensor surface and the pointer is controlled by the position control. A pointing stick measures the exerted force on the sensor surface and the pointer is controlled by the velocity control. Five subjects who were sufficiently accustomed to these devices volunteered for the experiment. The elapsed time for clicking all the circles was measured.

The typical fingerprint images during the operation are shown in Fig.17. Fig.17(a) (e) indicate the images at the position Fig.16(a) (e) respectively. Fig.17(f) indicates the image at the neutral position. From these figures, we can confirm the subject successfully clicks the target circles.

The average time of five trials for each device is shown in Fig.18. The proposed device relatively takes more time than the other devices (approximately an additional 2.0[sec] $(16.7 \%)$ and $1.6[\mathrm{sec}](11.9 \%)$ on average in comparison with the touch pad and the pointing stick respectively). However, these experimental results do not indicate that the proposed device is in principle inferior to those conventional devices. The touch pads and the pointing sticks have been commercially produced and improved for many years. The fact that the difference between the proposed and the traditional devices is only $10-17 \%$ indicates the usefulness of the proposed method.

By using the proposed device, an operator can easily control the pointer by a slight motion because a human's fingertip is sensitive to a slippage. Additionally, the device occupies a small space and can be shared with a fingerprint verification system. As a human-machine interface system, the device has advantages in the use of portable devices, such as PDAs and mobile phones. Moreover, the proposed method can be applied to haptic devices and teleoperation. Improvements of the operationality and the application will be considered in future work.

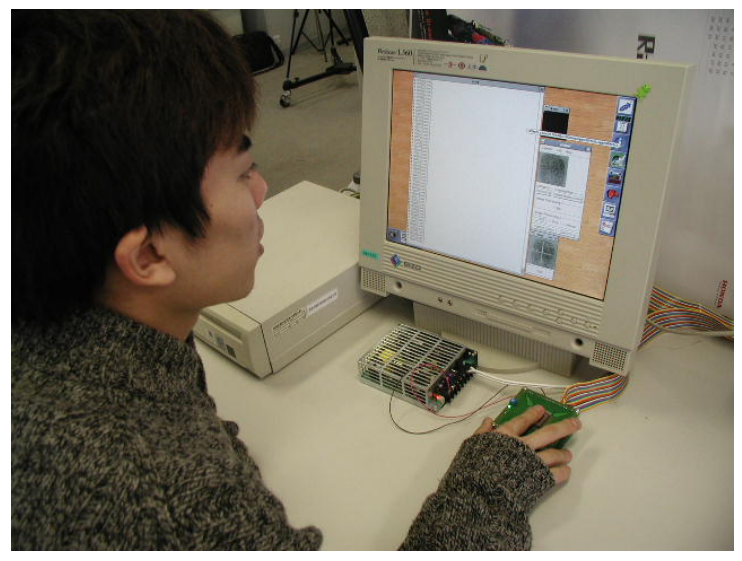

Fig. 15. Experimental scene. A subject operates the pointer on the screen by sliding the fingertip slightly on the sensor surface.

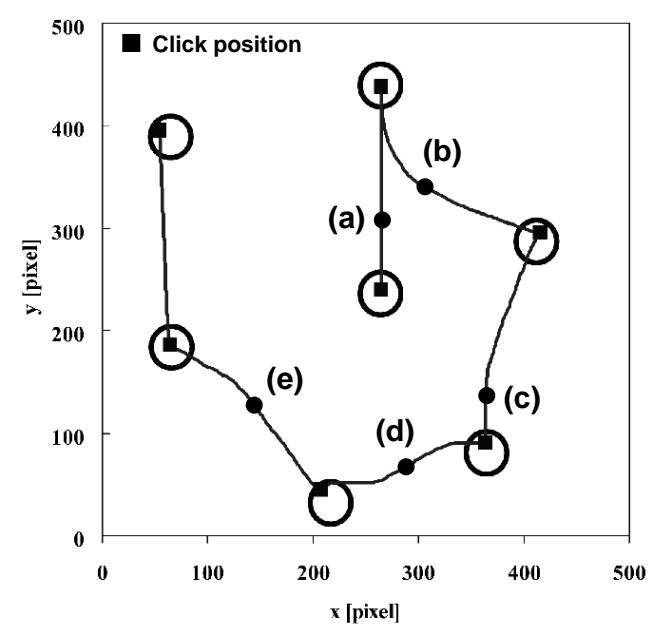

Fig. 16. Trajectory of the pointer during the operation. The operator sequentially moves the pointer to the circles on the screen and clicks.

\section{CONCLusion}

In this paper, a novel pointing device has been proposed that utilizes the deformation of the fingertip. The sliding motion before the fingertip is completely slipped is defined as a "semi-sliding motion". During the semi-sliding motion, an incipient slip occurs on the contact surface and a stick region remains around the center of the contact region. The velocity of the pointer is determined by detecting the center of the fingerprint and by calculating the eccentricity based on the deformation of the contact area. The center of the fingerprint is detected by the Group Delay Spectrum (GDS) tracking method. In order to investigate the correlation between the eccentricity and the sliding distance of the fingertip, and the correlation between the eccentricity and the exerted force on the sensor surface, a fingerprint measurement system has been constructed. The experimental results showed that the eccentricity has a linear correlation with the sliding distance of the fingertip. In order to confirm the effectiveness of the proposed method, a prototype of the pointing device system has been developed. The performance test has shown that 


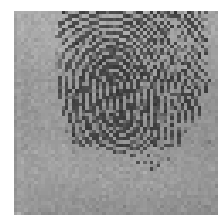

(a)

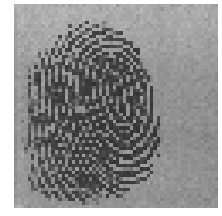

(d)

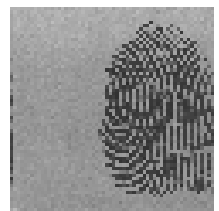

(b)

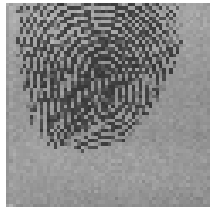

(e)

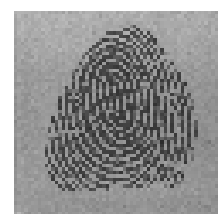

(c)

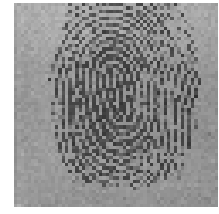

(f)
Fig. 17. Fingerprint images during the operation. (a) Up. (b) Right down. (c) Down. (d) Left down. (e) Left up. (f) Neutral.

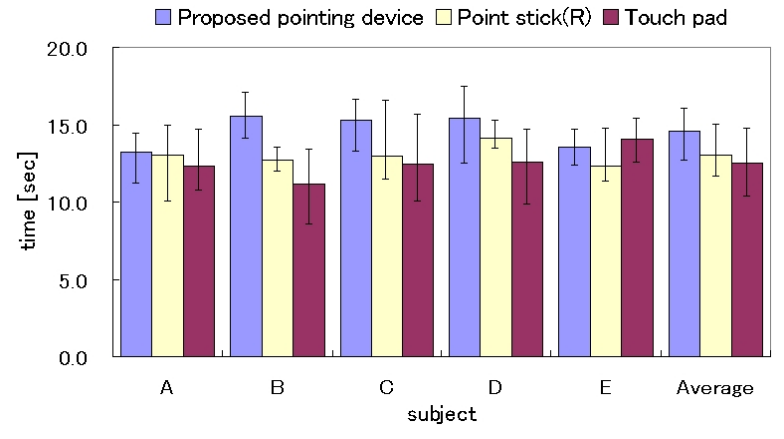

Fig. 18. Operation time for the performance test. The error bar means the standard deviation.

the subjects can successfully control the pointer using the developed device.

The proposed pointing device needs a smaller sensing surface than conventional devices and is suitable for portable devices. Additionally, the proposed device can be used for security devices by measuring the fingerprint information.

For the developed prototype, it is difficult to capture the fingerprint images when a fingertip was particularly sweaty or dry because we utilize an electric capacitance-type sensor. Recently, a new electric capacitance sensor, which is not largely effected by humidity, has become available. Moreover, other sensors, such as a pressure-sensitive and an optical-based type, are also commercially available.

The proposed method has the following problems and future work considers these problems: (1) The calculation time of the GDS should be reduced for better tracking. (2) Detection of the fingerprint center fails when the fingertip is remarkably rotated. (3) Investigation of the force estimation is not sufficient. (4) Improvement of the operationality and the application as a human-machine interface system.

\section{APPENDIX}

When the whole contact surface slips, the elastic object begins to completely slip over the rigid plate, which is called the gross slip. The partial slip that occurs before the gross slip is called the incipient slip. Hereafter, the contact region where a partial slip occurs is called as the slip region. The contact region where the objects surface is stuck is called as the stick region. The distance $c$ from the contact center to the boundary between the stick region and the slip region is given by:

$$
c=a(1-\phi)^{1 / 3}
$$

where $\phi \triangleq f_{l} / \mu f_{g}, f_{g}$ and $f_{l}$ are the normal and the tangential force, and $a$ is the radius of the contact area that is proportional to the normal force $f_{g}$ raised to the power of $1 / 3$ [34], [35].

When tangential force is applied on the object, the incipient slip makes the relative displacement $\delta_{x}$ shown in Fig.19. In this figure, $o$ and $o^{\prime}$ indicate the contact center before and after the slippage respectively. $\delta_{x}$ is derived from the following equation[36]:

$$
\delta_{x}=\frac{3 \mu f_{g}}{16 a}\left(\frac{2-\nu}{G}\right)\left\{1-\left(1-\phi^{2 / 3}\right)\right\}
$$

where $G=E / 2(1+\nu), \mu$ is the friction coefficient of the contact area, $\nu$ and $E$ are Poisson's ratio and Young's modulus of the elastic object.

From Eq.(18) and Eq.(19), both the distance $c$ and the relative displacement $\delta_{x}$ are functions of $\phi$. This indicates that the increase of the relative displacement $\delta_{x}$ correlates with the expanse of the incipient slip.

The relative displacement makes a deformation on the contact area $S_{d e f}$ shown in the meshed area of Fig.20. $S_{d e f}$ is derived from the following equation:

$$
S_{\text {def }}=\delta_{x} a \sin \psi+a^{2} \psi-\frac{\pi a^{2}}{2}
$$

where $\cos \psi=-\delta_{x} / a, \psi$ is the angle by x-axis and $o^{\prime}-o^{\prime \prime}$.

Since the area difference at the neutral position $S_{s x}$ is assumed 0 , the eccentricity along $\mathrm{x}$-axis $e_{x}$ is calculated from Eq.(1) and Eq.(20):

$$
\begin{aligned}
e_{x} & =\frac{S_{t x}}{S_{t}}-\frac{S_{s x}}{S_{s}} \\
& =\frac{\left(\frac{1}{2} \pi a^{2}+S_{d e f}\right)-\left(\frac{1}{2} \pi a^{2}-S_{d e f}\right)}{\pi a^{2}}-0 \\
& =\frac{2}{\pi}\left(\psi+\frac{\delta_{x}}{a} \sin \psi\right)-1 \\
& =\frac{2}{\pi}(\psi-\cos \psi \sin \psi)-1
\end{aligned}
$$

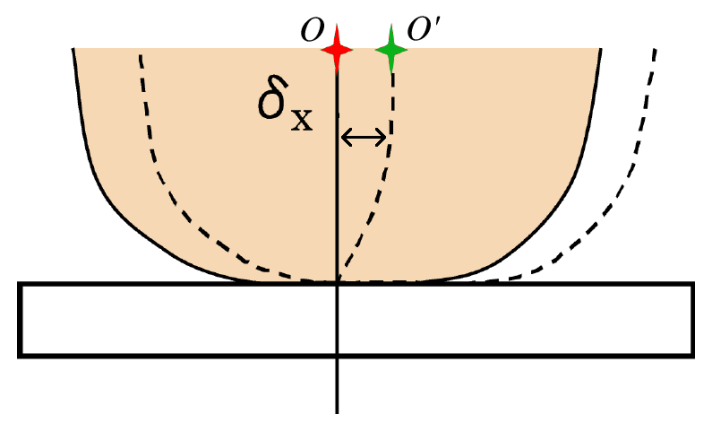

Fig. 19. Relative displacement of an elastic object by an incipient slip. 
If the displacement $\delta_{x}$ is significantly smaller than the radius of the contact $a$, we can also assume $\psi \cong-\cos \psi+$ $\pi / 2, \sin \psi \cong 1$. By substituting these approximation into Eq.(21), the following equation can be obtained:

$$
e_{x} \cong \frac{4 \delta_{x}}{\pi a}
$$

This equation indicates the eccentricity $e_{x}$ is in proportion to the sliding distance $\delta_{x}$.

\section{REFERENCES}

[1] C. Ware and H. Mikaelian. An evaluation of an eye tracker as a device for computer input. In Proceedings of the SIGCHI/GI conference on Human factors in computing systems and graphics interface, pages 183$188,1986$.

[2] R. Jacob. The use of eye movements in human-computer interaction techniques: what you look at is what you get. ACM Transactions on Information Systems, 9(2):152-169, 1991.

[3] J. Crowley, F. Berard, and J. Coutaz. Finger tracking as an input device for augmented reality. In International Workshop on Gesture and Face Recognition, 1995.

[4] F. Quek, T. Mysliwiec, and M. Zhao. Fingermouse : A freehand pointing interface. In Proceedings of the International Workshop on Automatic Face- and Gesture-Recognition, pages 372-377, 1995.

[5] M. Betke, J. Gips, and P. Fleming. The camera mouse: Visual tracking of body features to provide computer access for people with severe disablities. IEEE Transactions on Neural Systems and Rehabilitation Engineering, 10(1):1-10, 2002.

[6] I. Erdem, M. Erdem, Y. Yardimci, V. Atalay, and A. Cetin. Computer vision based mouse. In International Conference on Acoustics Speech and Signal Procedding, 2002.

[7] H. Cantzler and C. Hoile. A novel form of a pointing device. In Proceedings of Vision, Video and Graphics, pages 57-62, 2003.

[8] T. Zimmerman and J. Lanier. A hand gesture interface device. In Proceedings of CHI and Graphics Interface, pages 189-192, 1987.

[9] J. Perng, B. Fisher, S. Hollar, and K. Pister. Acceleration sensing glove. In The Third International Symposium on Wearable Computers, pages 178-180, 1999.

[10] M. Fukumoto and Y. Tonomura. Body coupled fingerring : wireless wearable keyboard. In Proceedings of the SIGCHI conference on Human factors in computing systems, pages 147-154, 1997.

[11] J. Rekimoto. Gesturewrist and gesturepad: Unobtrusive wearable interaction devices. In Fifth International Symposium on Wearable Computers, 2001.

[12] S. Mascaro and H. H. Asada. Virtual switch human-machine interface using fingernail touch sensors. In Proceedings of IEEE International Conference on Robotics and Automation, pages 2533-2538, 1999.

[13] K. L. Johnson. Contact Mechanics. Cambridge University Press, 1985.

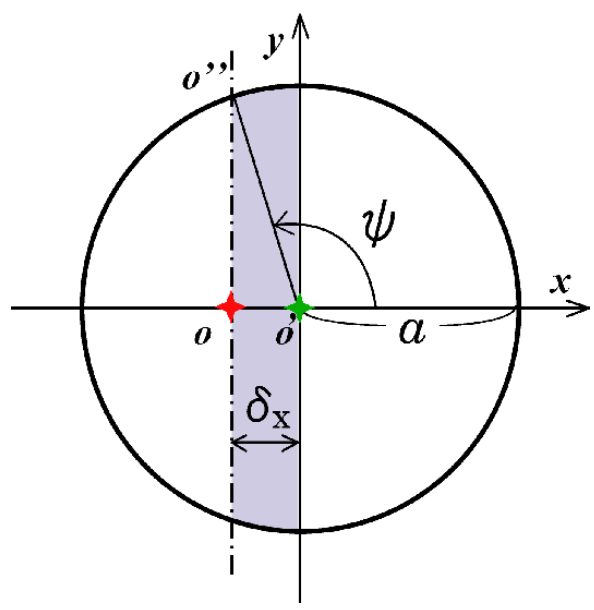

[14] M. Tada, T. Shibata, and T. Ogasawara. Investigation of the touch processing model in human grasping basedon the stick ratio within a fingertip contact interface. In Proceedings of IEEE International Conference on Systems, Man and Cybernetics, page tp1n4, 2002.10.

[15] R. S. Johansson and G. Westling. Roles of glabrous skin receptors and sensorimotor memory in automatic control of precision grip when lifting rougher or more slippery objects. Experimental Brain Research, 56:550-564, 1987.

[16] H. Shinoda and S. Ando. Ultrasonic emission tactile sensor for contact localization and characterization. In Proceedings of IEEE International Conference on Robotics and Automation, pages 2536-2543, 1994.

[17] N. Ferrier and W. Brockett. Reconstructing the shape of a deformable membrane from image data. The International Jornal of Robotics Research, 19(9):795-816, 2000.

[18] Y. Yamada, H. Morita, and Y. Umetani. Vibrotactile sensor generating impulsive signals for distinguishing only slipping states. In Proceedings of IEEE/RSJ International Conference on Intelligent Robots and Systems, pages 844-850, 1999.

[19] C. Melchiorri. Slip detection and control using tactile and force sensors. IEEE Transactions on Mechatronics, 5(3):235-243, 2000.

[20] Atsutoshi Ikeda, Yuichi Kurita, Jun Ueda, Yoshio Matsumoto, and Tsukasa Ogasawara. Grip force control for an elastic finger using visionbased incipient slip feedback. In Proceedings of IEEE/RSJ International Conference on Intelligent Robots and Systems, pages 810-815, 2004.

[21] M. Tremblay and M. Cutkosky. Estimating friction using incipien slip sensing during a manipulation task. In Proceedings of IEEE International Conference on Robotics and Automation, pages 429-434, 1993.

[22] K. Namamura and H. Shinoda. Tactile sensing device instantaneously evaluating friction coefficient. In Technical Digest of the 18th Sensor Symposium, pages 151-154, 2001

[23] S. Mascaro and H. H. Asada. Photoplethysmograph fingernail sensors for measuring finger forces without haptic obstruction. IEEE Transactions on Robotics and Automation, 17(5):698-708, 2001.

[24] S. Mascaro and H. H. Asada. Measurement of finger posture and threeaxis fingertip touch force using fingernail sensors. IEEE Transactions on Robotics and Automation, 20(1):26-35, 2004.

[25] M. Srinivasan, J. Whitehouse, and R. Lamotte. Tactile detection of slip: surface microgeometry and peripheral neural codes. Journal of Neurophysiology, 63(6):1323-1332, 1990.

[26] E. Serina, E. Mockensturm, C. Mote, and D. Rempe. A structual model of the forced compression of the fingertip pulp. Journal of Biomechanics, 31:639-646, 1998.

[27] T. Maeno and K. Kobayashi. Fe analysis of the dynamic characteristics of the human finger pad in contact with objects with/without surface roughness. In Proceedings of ASME International Mechanical Engineering Congress and Exposition, volume 64, pages 279-286, 1998.

[28] D. Pawluk and R. Howe. Dynamic contact of the human fingerpad against a flat surface. Journal of Biomechanical Engineering, 121:605$611,1999$.

[29] D. Maltoni, D. Mario, A. Jain, and S Prabhakar. Handbook of Fingerprint Verification. Springer, 2003.

[30] K. Eieccion. Automatic fingerprint identification. IEEE Spectrum, 10:36, 1973.

[31] Y. He, R. Kohno, and H. Imai. A fast automatic fingerprint identification approach based on a weighted-mean of binary image. IEICE Transactions on Fundamentals of Electronics, 76:1469-1482, 1993.

[32] H. Fujiyoshi, T. Umezaki, H Takeuchi, S. Saburi, and N. Suzumura. Fingerprint verification method using the transition probability of spectrum (in japanese). IEICE transactions on Information and Systems, J80-D-II(5): 1169-1177, 1997.

[33] N. Matsumoto, S. Sato, H. Fujiyoshi, and T. Umezaki. Evaluation of a fingerprint verification method based on lpc analysis. (in japanese). Transactions of IEE Japan, 122-C(5):799-807, 2002.

[34] R. D. Mindlin. Compliance of elastic bodies in contact. ASME Journal of Applied Mechanics, 16-E:259-268, 1949.

[35] N. Xydas and I. Kao. Modeling of contact mechanics and friction limit surface for soft fingers in robotics with experimental results. The International Jornal of Robotics Research, 18(8):941-950, 1999.

[36] S. Timoshenko and J.N. Goodier. Theory of Elasticity. McGraw-Hill Education, 1970

Fig. 20. Deformation of the contact area. 


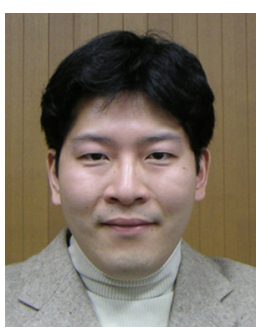

Yuichi Kurita received the B.E. degree from Osaka University, Osaka, Japan, in 2000, and the M.E and Ph.D. degree from Nara Institute of Science and Technology (NAIST), Nara, Japan, in 2002 and 2004 respectively all in information science. In 2005, he joined Hiroshima University, Hiroshima, Japan, and is currently a research associate of Robotics Laboratory in the Graduate School of Engineering. His research interests include human modeling and dextrous manipulation.

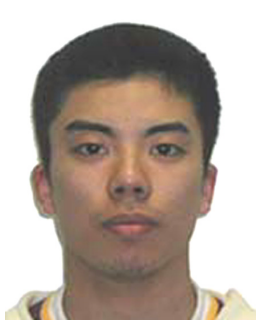

Atsutoshi Ikeda received the B.E. degree from Kagawa University, Kagawa, Japan, in 2002, and the M.E. degree from Nara Institute of Science and Technology (NAIST), Nara, Japan, in 2004. In 2004, he joined Yamaha Motor Co., LTD., Japan.

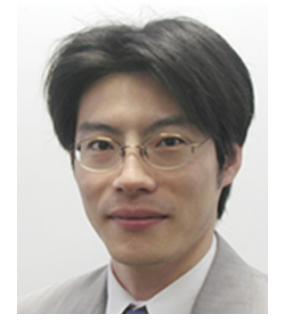

human modeling.
Jun Ueda received the B.S., M.S., and Ph.D. degree from Kyoto University, Kyoto, Japan, in 1994, 1996, and 2002 respectively all in mechanical engineering. From 1996 to 2000, he has been a research engineer of Advanced Technology R\&D Center, Mitsubishi Electric Corporation, Japan. In 2002, he joined Nara Institute of Science and Technology (NAIST), Nara, Japan, and is currently an Assistant Professor of Robotics Laboratory in the Graduate School of Information Science. His research interests include vibration control, robust control, manipulation, and

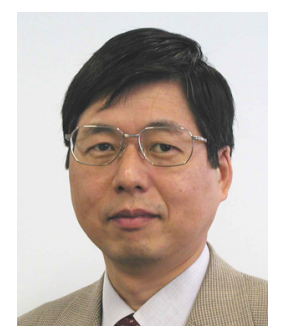

Tsukasa Ogasawara received the B.E., M.E., and $\mathrm{Ph} . \mathrm{D}$. degrees from The University of Tokyo, Tokyo, Japan, in 1978, 1980, and 1983, respectively. From 1983 to 1998 , he was with the Electorotechnical Laboratory, Ministry of International Trade and Industry, Japan. From 1993 to 1994, he was with the Institute for Real-Time Computer Systems and Robotics, University of Karlsruhe, Germany, as a Humboldt Research Fellow. In 1998, he joined Nara Institute of Science and Technology (NAIST), Nara, Japan, and is currently a Professor of Robotics Laboratory in the Graduate School of Information Science. His research interests include human-robot interaction, dexterous manipulation, and biologically inspired robotics. 